\title{
X-ray wavefront analysis and optics characterization with a grating interferometer
}

\author{
Timm Weitkamp, ${ }^{\text {a) }}$ Bernd Nöhammer, Ana Diaz, and Christian David \\ Laboratory for Micro- and Nanotechnology, Paul Scherrer Institut, Villigen, Switzerland \\ Eric Ziegler \\ Experiments Division, European Synchrotron Radiation Facility, Grenoble, France
}

(Received 5 May 2004; accepted 17 December 2004; published online 24 January 2005)

\begin{abstract}
We present an interferometric method to measure the shape of a hard-x-ray wavefront. The interferometer consists of a phase grating as a beam splitter and an absorption grating as a transmission mask for the detector. The device can be used to measure wavefront shape gradients corresponding to radii of curvature as large as several dozens of meters, with a lateral resolution of a few microns. This corresponds to detected wavefront distortions of approximately $10^{-12} \mathrm{~m}$ or $\lambda / 100$. The device was used with $12.4 \mathrm{keV} \mathrm{x}$ rays to measure the slope error and height profile of an $\mathrm{x}$-ray mirror. Surface slope variations with periods ranging from less than $1 \mathrm{~mm}$ to more than 1 $\mathrm{m}$ can be detected with an accuracy better than $0.1 \mu \mathrm{rad}$. (C) 2005 American Institute of Physics. [DOI: $10.1063 / 1.1857066$ ]
\end{abstract}

Modern synchrotron radiation (SR) sources are highly brilliant, and their radiation has a considerable degree of transverse coherence. $\mathrm{X}$ rays from these sources can therefore be focused into spots much smaller and more intense than previously possible, which has led to tremendous advances in x-ray microanalysis. The coherence properties of unfocused radiation are also exploited in techniques such as various methods of phase-sensitive radiography ${ }^{1}$ and coherent scattering. ${ }^{2,3}$ But the performance of those methods and, more generally, of any setup relying on well-defined illumination can be strongly degraded by uncontrolled distortions of the x-ray wavefront. Beam quality may be affected in this way by such things as roughness or longer-range shape errors of reflective or transmitting surfaces, misalignment of components, or a miscalibration of adaptive elements such as bendable mirrors or crystals.

While highly sensitive wavefront measurement techniques for soft $\mathrm{x}$ rays and extreme ultraviolet radiation have been developed in the past few years, ${ }^{4}$ few wavefront analysis methods for hard $\mathrm{x}$ rays are available that allow the measurement of wavefront curvature and distortions. Noninterferometric methods are successfully applied in in-line phasecontrast microradiography, ${ }^{5}$ but they are not very sensitive to long-range transverse phase variations, such as those that correspond to overall divergence. Crystal interferometers ${ }^{6,7}$ yield direct phase information, but their fabrication is demanding, and they are highly sensitive to vibration and mechanical drift.

In this letter, we present a robust grating interferometer for hard $\mathrm{x}$ rays that allows wavefront analysis with high sensitivity. We further demonstrate the use of this instrument for the analysis of the wavefront downstream of an x-ray mirror and the surface characterization of the mirror.

Figure 1 shows the principle of the interferometer used in this study. A Ronchi phase grating splits the incident beam into essentially two partial waves with a small shear angle $\vartheta_{\mathrm{s}}=2 \lambda / d_{1}$ (with $\lambda$ as the wavelength and $d_{1}$ as the period of

${ }^{a)}$ Electronic mail: timm.weitkamp@psi.ch the grating). At a distance $L$ behind the phase grating, the two waves with a lateral shear $s=\vartheta_{\mathrm{s}} \times L$ will form an interference pattern, provided the transverse coherence length $\ell_{c}$ at the position of the grating is sufficient, i.e., $\ell_{\mathrm{c}} \gtrsim s$. If the incident wavefront is an unperturbed plane wave, then this pattern is a set of parallel straight interference fringes with a period that equals half the period of the phase grating. Perturbations of the wavefront will distort these interference fringes. The principle of the method presented here is to detect the fringe distortion and thus obtain information on the wavefront perturbation. In order for the shear $s$ to be sufficiently large at hard x-ray wavelengths, the period $d_{1}$ of the phase grating must not exceed a few microns. The interference fringes will then have a spacing in the micron range, which is beyond the resolution of most area detectors, especially of those with a reasonably large field of view. This problem can be solved by an absorption grating with pitch $d_{2}=d_{1} / 2$ placed in the detection plane. If the two gratings are tilted with respect to each other about the optical axis by a small angle $\alpha$, the intensity distribution immediately downstream of the absorption grating is a moire pattern of the interference pattern incident on the absorption grating with the lines of that grating. Note that here the absorption grating is not used as a diffractive element but only as a transmission mask. In the plane-wave case, the moiré fringes have a spacing $d_{m}=d_{2} /[2 \sin (\alpha / 2)] \approx d_{2} / \alpha$, which is much larger than $d_{2}$ and can be easily tuned by varying the tilt angle $\alpha$. The

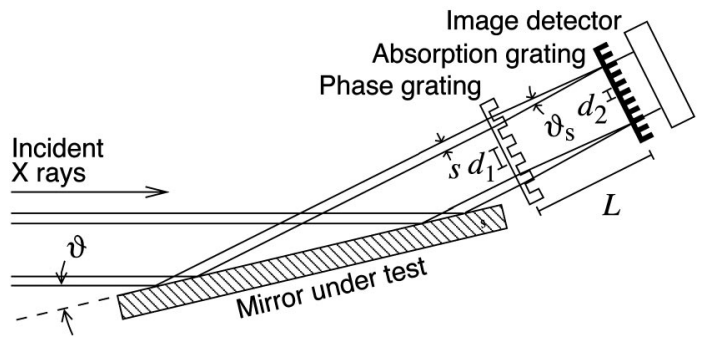

FIG. 1. Setup for measurement of a wavefront distorted by an optical component (mirror) with an interferometer consisting of a phase grating as a beam splitter and an absorption grating as an analyzer. 


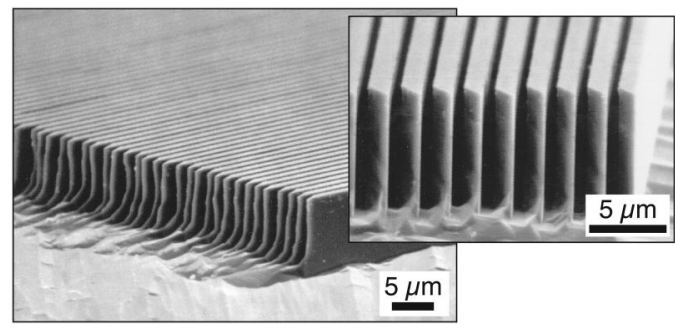

FIG. 2. Scanning electron micrographs of the phase grating used in the interferometer.

moiré fringes can then be resolved on a conventional area detector as used in microradiography.

The gratings for the interferometer were fabricated in a similar way as those of a less compact device presented earlier. ${ }^{8}$ The linear phase grating is made of silicon and has a pitch of $d_{1}=2 \mu \mathrm{m}$ and a thickness of $9 \mu \mathrm{m}$. It was fabricated by electron-beam lithography and anisotropic wet etching into silicon. ${ }^{9}$ Figure 2 shows an electron micrograph of the phase grating. For the fabrication of the absorption grating, the same process was used to make a silicon grating of pitch $d_{2}=1 \mu \mathrm{m}$. Gold was then evaporated on this grating at an incidence angle chosen such that the metal accumulated mainly on one side of the silicon side walls. As in the earlier interferometer, ${ }^{8}$ the effective thickness of the phase grating was increased to a phase shift of $\pi$ (and thus to optimum efficiency, eliminating the zeroth diffraction order) by mounting both gratings with a tilt about the axis perpendicular to the optical axis and the lines of the grating.

The interferometer was used to measure the wavefront shape behind a multilayer mirror and determine the surface profile of the mirror. The measurements were carried out at the bending-magnet beamline BM 5 of the European Synchrotron Radiation Facility (ESRF). A photon energy of 12.4 $\mathrm{keV}$, i.e., $\mathrm{x}$-ray wavelength of $\lambda=1.00 \AA$, was selected by a silicon double-crystal monochromator. The coherent beam cross section at the experimental setup was $15 \times 50 \mu \mathrm{m}(H$ $\times V$ ). The object under test was a $150-\mathrm{mm}$-long W/Si flat multilayer mirror with a Bragg angle of $\vartheta=13.5 \mathrm{mrad}$ at $12.4 \mathrm{keV}$. The mirror was mounted horizontally so that the $\mathrm{x}$-ray beam was deflected upwards (Fig. 1). The gratings of the interferometer were mounted with their lines horizontal. The phase grating was placed $50 \mathrm{~mm}$ away from the downstream edge of the mirror. The inter-grating distance was $L$ $=85 \mathrm{~mm}$, a setting where the fractional Talbot effect gives good fringe visibility and which corresponds to a vertical shear of $s=L \times \vartheta_{s}=2 L \lambda / d_{1}=8.5 \mu \mathrm{m}$.

The moire intensity distribution behind the analyzer grating was recorded using an imaging detector with a field of view of $3.5 \times 3.5 \mathrm{~mm}^{2}$. Since the longitudinal position on the mirror surface, corresponding to the vertical position in the detector plane, is squeezed in the images by a factor of $\vartheta$, these settings allowed the entire longitudinal extent of the mirror to fit in the field of view of the detector. The detector was placed closely behind the absorption grating $(\approx 10 \mathrm{~mm})$ so that the unwanted effect of diffraction by this grating could be neglected. The flat beam without the mirror had been measured before to exclude other sources of wavefront distortion.

A region of interest of an interferogram obtained in this setup, with an exposure time of $10 \mathrm{~s}$, is shown in Fig. 3(a). The visibility of the fringes ranges between 3 and 5\%, and
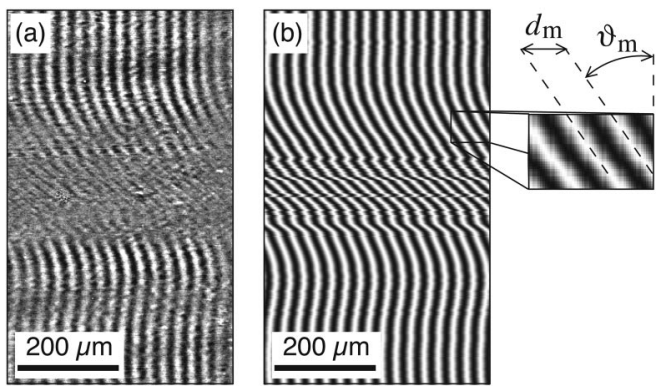

FIG. 3. Detail of an interferogram (a) and reduced image containing only relevant fringes (b). Fringe spacing $d_{\mathrm{m}}$ and inclination $\vartheta_{\mathrm{m}}$ are used in the analysis of the pattern.

the median contrast-to-noise ratio is 8.0. The observed moiré fringes lie nearly parallel to the projected longitudinal mirror-position coordinate. Shape errors along the longitudinal direction affect the horizontal position of the moirés.

A simple method of fringe analysis consists in the determination of moiré-fringe frequency and position (i.e., fringe phase) from an ensemble of fringes over many pixel columns of the detector, but independently for each horizontal pixel row. In this case, horizontal sections 250 pixels $(440 \mu \mathrm{m})$ wide and one pixel high were analyzed using Fourier methods. Frequency and fringe positions determined in this way are shown in Fig. 3(b). The local radius of curvature $R$ of the wave along the vertical direction can then be determined from the moiré-fringe inclination angle $\vartheta_{\mathrm{m}}$ using the relation ${ }^{10}$

$$
R=\frac{d_{\mathrm{m}}}{d_{2}} L \tan \vartheta_{\mathrm{m}} .
$$

Figure 4 shows a map of $R$ obtained in this way for the entire reflected beam. Several detector shots at different horizontal detector positions were patched together for this. The moiré fringe position along the horizontal coordinate is proportional to the longitudinal slope error $\delta$ of the mirror and is related to it by

$$
\delta=\Phi \times d_{2} /(4 \pi L),
$$

where $\Phi$ is the fringe phase, or normalized horizontal fringe position $\Phi=2 \pi \times x_{m} / d_{m}$ (with $x_{m}$ as the horizontal fringe position and $d_{m}$ as the horizontal fringe spacing). The height profile $h(l)$ along the longitudinal mirror coordinate $l$ is then obtained by integration: $h(l)=\int_{0}^{L} \delta(l) d l$. Figure 5 shows the reconstructed slope error and height profile of the same mirror region as depicted in Fig. 3. The bent profile is plausible

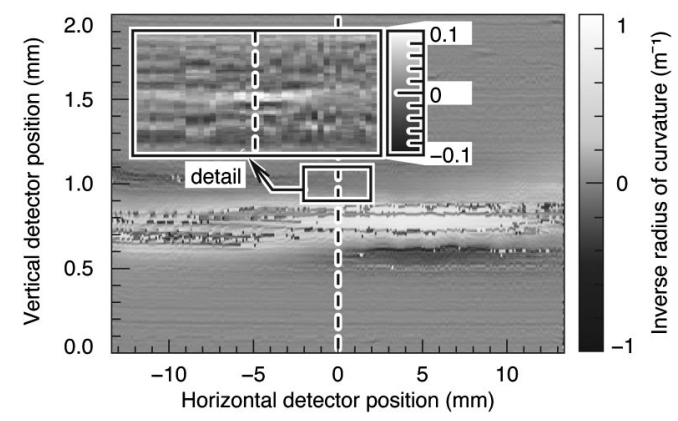

FIG. 4. Map of the local wavefront curvature radius along the vertical direction. The dashed line represents the position for which the mirror slope profile is shown in Fig. 5. 


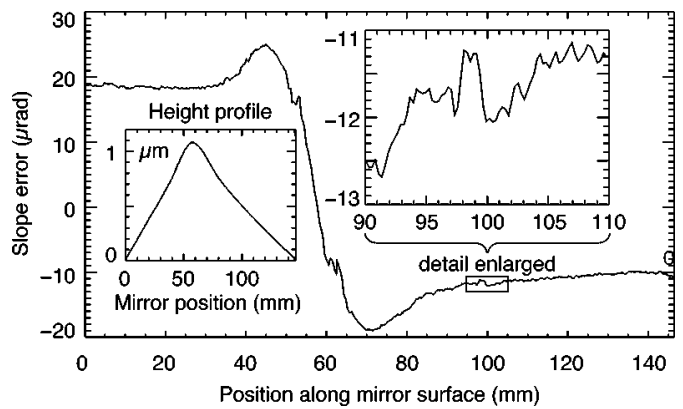

FIG. 5. Reconstructed slope error (main curve and lower-right inset) and height profile (upper-left inset) along the longitudinal coordinate of the mirror surface.

given that the mirror was provisionally mounted with wax on a central support.

The resolution along the vertical position of the detector, corresponding to the position along the mirror surface, is limited both by the vertical shear $s$ and by the effective vertical pixel size. In the present case, the shear is the predominant limitation and limits the resolution to approximately the value of $s$, i.e., $8.5 \mu \mathrm{m}$, or $\Delta l \approx s / \vartheta=0.65 \mathrm{~mm}$ in terms of mirror-surface position. The resolution in horizontal direction is limited by the choice of the number of pixel columns used for the moiré fringe analysis. In this example where a relatively wide region was used, the resolution is approximately $0.5 \mathrm{~mm}$. Using more elaborate analysis methods, the resolution may be optimized to the order of one moiré-fringe period.

The accuracy of the slope error is limited by the accuracy to which the moiré phase $\Phi$ is determined. An estimated value for this accuracy can be obtained directly from the experimental data: The average fluctuations of fringe phase between adjacent pixel rows are less than 1/50 of a moiré period. Since the phase determination for each pixel row is completely independent of other rows, these fluctuations include all statistical contributions. The uncertainty of the determined fringe phase is therefore $\Delta \Phi<2 \pi / 50$. This results in an uncertainty of the slope error of $\Delta \delta<\Delta \Phi \times d_{2} /(2 L)$ $\approx 74 \mathrm{nrad}$. The corresponding mechanical stability requirement is a relative transverse movement of the two gratings of less than $d_{2} / 50=20 \mathrm{~nm}$, which is easily fulfilled over long times. The maximum radius of curvature that can be detected is on the order of $R_{\max }=(\Delta \Phi / 2 \pi) s^{2} / \lambda \approx 35 \mathrm{~m}$. In terms of path-length difference across the resolution length $s$, this corresponds to $s^{2} / 2 R_{\max }=10^{-12} \mathrm{~m}$. These characteristics meet metrology needs for modern SR optics. ${ }^{11}$ The sensitivity can be further increased by choosing a larger intergrating distance $L$ or smaller grating periods $d_{1}$ and $d_{2}$, both resulting in a larger shear $s$, at the expense of lateral resolution. The instrument is light and compact and can easily be installed as long as the x-ray beam is accessible directly downstream of the optic under test. We therefore believe that this interferometer is suitable for routine use in in situ, at-wavelength $\mathrm{x}$-ray mirror metrology and performance monitoring and, more generally, for various $\mathrm{x}$-ray wavefront analysis tasks as well as phase-contrast imaging.

The authors would like to thank the technical staff at PSI for assistance in the fabrication of the gratings, beamline staff at BM 5 for assistance during the experiment, and A. Somogyi of ID 22 at ESRF for loan of equipment. The multilayer mirror was manufactured by P. Høghøj at the Institut Laue-Langevin in Grenoble. Three of the authors (A.D., B.N., and T.W.) received support from the Swiss National Science Foundation, the Swiss Federal Office for Education and Science, and the European Union.

${ }^{1}$ R. Fitzgerald, Phys. Today 53, 23 (2000).

${ }^{2}$ J. Miao, P. Charalambous, J. Kirz, and D. Sayre, Nature (London) 400, 342 (1999).

${ }^{3}$ I. K. Robinson, I. A. Vartanyants, G. J. Williams, M. A. Pfeifer, and J. A. Pitney, Phys. Rev. Lett. 87, 195505 (2001).

${ }^{4}$ P. Naulleau, K. A. Goldberg, E. H. Anderson, P. Batson, P. E. Denham, K. H. Jackson, E. M. Gullikson, S. Rekawa, and J. Bokor, J. Vac. Sci. Technol. B 19, 2396 (2001).

${ }^{5}$ P. Cloetens, W. Ludwig, J. Baruchel, D. V. Dyck, J. V. Landuyt, J. P. Guigay, and M. Schlenker, Appl. Phys. Lett. 75, 2912 (1999).

${ }^{6} \mathrm{U}$. Bonse and M. Hart, Appl. Phys. Lett. 6, 155 (1965).

${ }^{7}$ A. Momose, Opt. Express 11, 2303 (2003).

${ }^{8}$ C. David, B. Nöhammer, H. H. Solak, and E. Ziegler, Appl. Phys. Lett. 81, 3287 (2002)

${ }^{9}$ C. David, E. Ziegler, and B. Nöhammer, J. Synchrotron Radiat. 8, 1054 (2001).

${ }^{10}$ T. Weitkamp, A. Diaz, B. Nöhammer, F. Pfeiffer, M. Stampanoni, E. Ziegler, and C. David, Proc. SPIE 5533, 140 (2004).

${ }^{11}$ L. Assoufid, O. Hignette, M. Howells, S. Irick, H. Lammert, and P. Takacs, Nucl. Instrum. Methods Phys. Res. A 467, 267 (2001). 